\title{
How-to: Write a parton-level Monte Carlo particle physics event generator
}

\author{
Andreas Papaefstathiou, \\ PH Department, TH Department, CERN, CH-1211 Geneva 23, Switzerland \& Higgs Centre for \\ Theoretical Physics, University of Edinburgh, Peter Guthrie Tait Road, Edinburgh EH9 3FD, UK. \\ E-mail: apapaefs@cern.ch
}

\begin{abstract}
This article provides an introduction to the principles of particle physics event generators that are based on the Monte Carlo method. Following some preliminaries, instructions on how to build a basic parton-level Monte Carlo event generator for the hard interaction are given through exercises. Indications on how to proceed to full event simulations are given. ${ }^{1}$
\end{abstract}

\footnotetext{
${ }^{1}$ The related course was given as part of the "Advanced Scientific Computing Workshop" at ETH Zürich in July 2014.
} 


\section{Contents}

$\begin{array}{ll}\text { Preface } & 1\end{array}$

1 Introduction $\quad 2$

2 Preliminaries 3

2.1 Monte Carlo integration 3

2.2 Improving convergence of the Monte Carlo integration 4

2.3 Hit-or-Miss Monte Carlo 5

$\begin{array}{lll}2.4 & \text { Factorisation and the structure of event generators } & 6\end{array}$

3 Exercises $\quad 8$

3.1 Particle physics input $\quad 9$

3.1.1 $e^{+} e^{-} \rightarrow \gamma \rightarrow \mu^{+} \mu^{-} \quad 9$

3.1.2 $e^{+} e^{-} \rightarrow Z / \gamma \rightarrow \mu^{+} \mu^{-} \quad 12$

3.2 Exercise 1: lepton colliders 13

$\begin{array}{lll}3.3 & \text { Exercise 2: hadron colliders } & 13\end{array}$

4 After the hard process $\quad 16$

$\begin{array}{lll}5 & \text { Conclusions } & 17\end{array}$

$\begin{array}{llr}6 & \text { Acknowledgements } & 17\end{array}$

$\begin{array}{ll}\text { A Constants } & 17\end{array}$

$\begin{array}{ll}\text { B Parton density functions using LHAPDF } & 17\end{array}$

C The Les Houches event file format $\quad 18$

$\begin{array}{lr}\text { D Convergence } & 19\end{array}$

\section{Preface}

According to Wikipedia, "a how-to is an informal, often short, description of how to accomplish a specific task. A how-to is usually meant to help non-experts, may leave out details that are only important to experts, and may also be greatly simplified from an overall discussion of the topic." [1]. In some aspects this is also valid for this article. However, in this case the aim of this article is to provide some insight to the experts themselves, that is, physicists, who may use Monte Carlo event generators as "black boxes" to serve their purposes, either to calculate cross sections or to generate events to further simulate and investigate a future possible experimental analysis. 


\section{Introduction}

Treatment of particle collisions in mechanics starts off relatively easy: we initially study elastic collisions of two spheres in one dimension, and we are asked to calculate the various momenta after a collision occurs. The next complication involves adding the effects of inelasticity. This results in some energy loss e.g. through the balls sticking together and so on. The theoretical description of collisions of elementary particles starts off equally simply: the scattering of two electrons, for example, can be simulated at the first order in the perturbative picture (leading order), via the exchange of a single photon, representing an elastic collision. However, "Truth is stranger than Fiction, but it is because Fiction is obliged to stick to possibilities; Truth isn't." [2]. In the context of particle physics, to describe 'Truth', i.e. Nature, in our 'fictional' simulations, we need to model a multitude of effects using a series of approximations and models. To name but a few of these aspects:

- particles radiate, e.g. photons off electrons, gluons off quarks,

- incoming particles may be confined in a bound state, e.g. quarks and gluons in protons,

- higher-order corrections in perturbation theory are too laborious to compute beyond the first few orders,

- the phase space of the final-state particles is huge and of a variable number of dimensions,

- and many effects cannot be described by perturbation theory and need to be modelled.

Many of the above effects have been incorporated into computer simulations using Monte Carlo techniques.

The large dimensionality of the phase space makes the Monte Carlo integration the method of choice. The Markovian nature of the parton shower process can also be formulated as a Monte Carlo process. For different aspects of the simulation, several tools already exist on the "market". These serve many purposes, sometimes overlapping, following different approaches and methodologies. Without (and far from) being completely inclusive, some of these tools are (i) MadGraph, (ii) HERWIG 7, (iii) Pythia 8 and (iv) Sherpa. MadGraph provides parton-level events of automatically generated process that the user asks for, at the moment capable of generating events at leading order and next-to-leading order in QCD (via the MC@NLO method). The output can then be given to a general-purpose event generator for showering and hadronization $[3,4]$. HERWIG $7[5-11]^{1}$, Pythia $8[12,13]$ and Sherpa [14] are general-purpose event generators that include in part some automation for generating processes at parton level as well as taking into account the effects of the parton shower, hadronization and the underlying event.

For a review of the detailed physics and the philosophy behind Monte Carlo event generators, I refer the reader to Ref. [15]. Here we wish to examine the minimal aspects of

\footnotetext{
${ }^{1}$ Formerly known as HERWIG++.
} 
constructing a parton-level event generator, adding some hints at the end for how one can incorporate the more advanced features such as a parton shower, hadronization, the underlying event and including higher-order corrections. We will start with some preliminaries in the next section.

\section{Preliminaries}

\subsection{Monte Carlo integration}

This section has been adapted in part from Peter Richardson's CTEQ 2006 lectures $^{2}$ as well as Mike Seymour's PhD thesis, Chapter $3 .^{3}$

Monte Carlo integration is based on a simple observation: the value of an integral can be recast as the average of the integrand:

$$
I=\int_{x_{1}}^{x_{2}} \mathrm{~d} x f(x)=\left(x_{2}-x_{1}\right)\langle f(x)\rangle .
$$

Consequently, this implies that if we take some, say $N$, values of $x$, distributed uniformly in $\left(x_{1}, x_{2}\right)$, then the average of $f(x)$ will be a good estimator of the integral, $I$. We can then write:

$$
I \approx\left(x_{2}-x_{1}\right) \frac{1}{N} \sum_{i=1}^{N} f\left(x_{i}\right) .
$$

We can draw the values $x_{i}$ randomly: if $\rho_{i}$ is a uniform random number in $(0,1),{ }^{4}$ then we have:

$$
x_{i}=\left(x_{2}-x_{1}\right) \rho_{i}+x_{1} .
$$

To estimate the accuracy of the calculation we can employ the Central Limit Theorem: the distribution of $\langle f(x)\rangle$ will tend to a Gaussian with standard deviation $\sigma_{\mathrm{MC}}=\sigma / \sqrt{N}$, where $\sigma$ is the standard deviation of $f\left(x_{i}\right)$. Our inaccuracy simply decreases as $1 / \sqrt{N}$. We often also define the weight: $W_{i}=\left(x_{2}-x_{1}\right) f\left(x_{i}\right)$, and then the integral is simply the average of the weight:

$$
I \approx I_{N}=\frac{1}{N} \sum_{i=1}^{N} W_{i} .
$$

We also define the variance, $V_{N} \equiv \sigma^{2}$ :

$$
V_{N}=\frac{1}{N} \sum_{i} W_{i}^{2}-\left[\frac{1}{N} \sum_{i} W_{i}\right]^{2},
$$

from which $\sigma_{\mathrm{MC}}=\sqrt{V_{N} / N}$, and we finally arrive at the expression:

$$
I \approx I_{N} \pm \sqrt{\frac{V_{N}}{N}}
$$

\footnotetext{
${ }^{2}$ http://www.ippp.dur.ac.uk/ richardn/talks/.

${ }^{3}$ http://www.hep.manchester.ac.uk/u/seymour/thesis/.

${ }^{4}$ That is, with equal probability to lie anywhere within the given interval.
} 
One can compare the convergence of the Monte Carlo integration technique to those for other common techniques. In $d$-dimensions the convergence of techniques such as the 'Trapezium Rule', 'Simpson's rule' and Gaussian quadrature are $\propto 1 / N^{2 / d}, \propto 1 / N^{4 / d}$ and $1 / N^{(2 m-1) / d}$ respectively. On the other hand, Monte Carlo integration always extends trivially and converges as $\propto 1 / \sqrt{N}$ in $d$ dimensions, and hence converges already faster than all the aforementioned methods in $d>4, d>8$ and $d>4 m-2$ respectively. ${ }^{5}$ In typical LHC events we have $\mathcal{O}(1000)$ particles and hence this results in $O(3000)$ phase space integrals. Therefore, Monte Carlo integration is in fact the only viable option.

The biggest disadvantage of the Monte Carlo method is the relatively slow divergence in few dimensions. This can be tackled by 'Importance Sampling', which we will discuss below. Its principal advantages over numerical quadrature can be summarised as:

- fast convergence in many dimensions,

- arbitrarily complex integration regions,

- small feasibility limit: the minimum number of functional evaluations which must be made for the method to work at all, in this case 2,

- small growth rate: the smallest number of additional function evaluations needed to improve the current estimate, in this case 1: each additional point improves the estimate of the integral,

- easy estimate of accuracy.

\subsection{Improving convergence of the Monte Carlo integration}

The accuracy of an integral calculated via the Monte Carlo integration method is given by $\sqrt{V_{N} / N}$. Thus one can simply increase the number of points to increase the accuracy. However, one can also look for ways to decrease $V_{N}$, e.g., by a method called 'Importance Sampling' [16]. The basic idea is to perform a Jacobian transform so that the integral is flatter in the new integration variable. This is equivalent to finding a transform such that $V_{N}^{\prime}<V_{N}$.

We begin by considering the simplest case encountered in particle physics. In cross section calculations we often encounter the so-called Breit-Wigner distribution, that describes the 'peak' of a resonance:

$$
F_{\mathrm{BW}}\left(m^{2}\right)=\frac{1}{\left(m^{2}-M^{2}\right)^{2}+M^{2} \Gamma^{2}},
$$

where $M$ would be the physical (on-shell) mass of the particle, $m$ is the off-shell mass and $\Gamma$ its width. An example of the distribution (made using $M=90, \Gamma=10$ ) is shown in Fig. 1.

We then often encounter integrals of the form:

$$
I=\int_{M_{\min }^{2}}^{M_{\max }^{2}} \mathrm{~d} m^{2} \frac{1}{\left(m^{2}-M^{2}\right)^{2}+M^{2} \Gamma^{2}} .
$$

\footnotetext{
${ }^{5} \mathrm{~A}$ summary of the rate of convergence of the various techniques is given in Table 3 in Appendix D.
} 


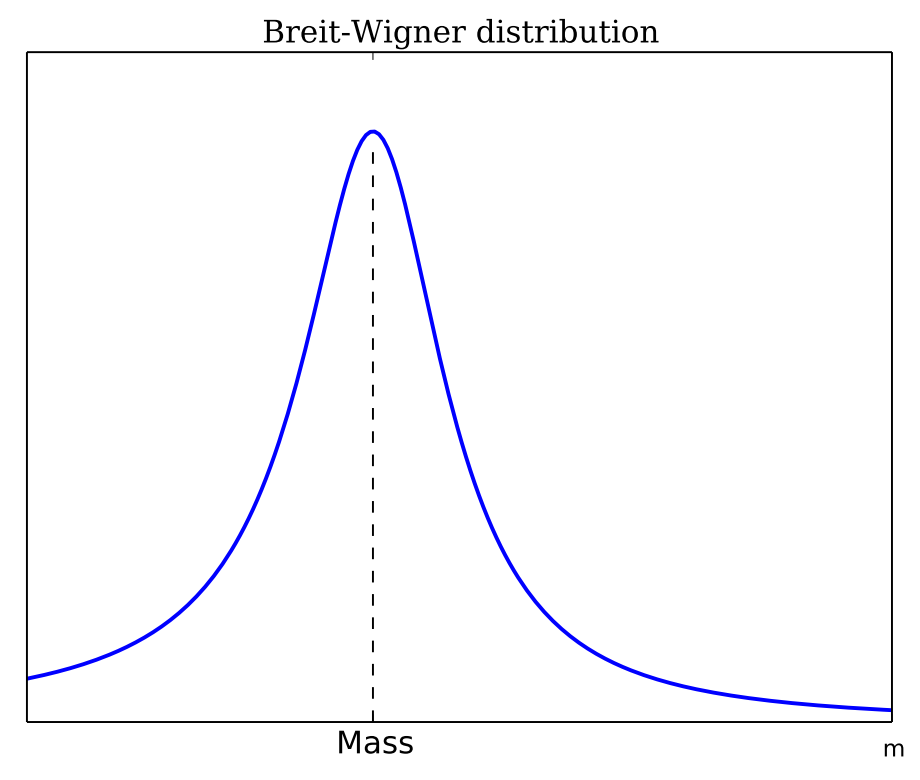

Figure 1: An example of the Breit-Wigner distribution, made for $M=90, \Gamma=10$.

The transformation we wish to consider is $m^{2} \rightarrow \rho$, where

$$
m^{2}=M \Gamma \tan \rho+M^{2},
$$

and the corresponding Jacobian is given by:

$$
J=\left|\frac{\partial m^{2}}{\partial \rho}\right|=M \Gamma \sec ^{2} \rho .
$$

Hence we have:

$$
\begin{aligned}
I & =\int_{\rho_{\min }}^{\rho_{\max }} \mathrm{d} \rho\left|\frac{\partial m^{2}}{\partial \rho}\right| \frac{1}{\left(m^{2}-M^{2}\right)^{2}+M^{2} \Gamma^{2}} \\
& =\frac{1}{M \Gamma} \int_{\rho_{\min }}^{\rho_{\max }} \mathrm{d} \rho .
\end{aligned}
$$

It is evident that in this case, we have in fact reduced the variance to zero: $V_{N}^{\prime}=0$. In practice, few of the cases we need to deal with can be exactly integrated. In cases of complicated integration regions, one can try and pick a function that approximates the behaviour of the function we want to integrate. A specific method, called multi-channel integration, aims to handle the situation where one is faced with multiple peaks in the phase space and one can then not use a single Breit-Wigner. The method can be automated and is used in all modern Monte Carlo event generators [17].

\subsection{Hit-or-Miss Monte Carlo}

There are two main aspects of Monte Carlo that make it ideal for use in constructing event generators: the close relationship between the numerical method and the physical process under study, both being 'random' in some sense, and the ability to make unweighted events. 
In a similar way that a Monte Carlo integration of the sort described in Section 2.1 is performed, one can scan over the function $f(x)$ and collect a set of phase-space points, along with their associated probabilities, corresponding to the weight of each in the integral. These points effectively correspond to possible 'events', with their weights corresponding to their probability of occurring. However, if we want to use these events, e.g. to perform an experimental analysis, then we must always carry the associated weight around for use in histograms, averages and so on. This can be inconvenient but also very inefficient: time may be wasted in some latter part of the simulation (e.g. detector simulation) to events that possess only a very small weight. The so-called 'hit-or-miss' method aims to equalize the weights of different events as far as possible.

Since the weight of each event is proportional to the probability of it occurring, we can unweigh the events by keeping only a fraction of them, according to their weights. We do this by finding the maximum weight which occurs in the integration region. This can be done while performing Monte Carlo integration. We choose to keep ('accept') each event with probability $f(x) / f_{\max }$. The rest are thrown away ('rejected'). All accepted events are given a weight $\langle f\rangle$, calculated from the Monte Carlo integral over all generated events (not just the accepted events). The complete algorithm for integration and event generation is then:

1. Monte Carlo integration and scanning are performed: $N$ points are picked randomly, according to some distribution and their weight is accumulated to the sums: $\sum_{i} W_{i}$, $\sum_{i} W_{i}^{2}$. The cross section and corresponding error are computed according to Eqs. 2.4 and 2.1. During this period, the phase-space point which give the maximum weight, $W_{\max }$ is stored.

2. Generating unweighted events via the 'hit-or-miss' method: go through randomly chosen phase-space points and compare the probability of each, given by $W_{i} / W_{\max }$ to a random number $R \in(0,1)$. If $W_{i} / W_{\max }>R$, we 'accept' the event, otherwise we reject it. This is done until we have collected the desired number of events, $N_{\text {events }}$.

\subsection{Factorisation and the structure of event generators}

The complexity of an event is something that we (particle physicists) are all familiar with. This is exemplified in Fig. 2. Even if the hard collision is simple, we expect thousands of final state particles at hadron colliders. It is evident that this poses many challenges in simulating events: it is difficult or even impossible to construct an efficient algorithm but also hard to exactly calculate final-state distributions of hadrons.

It is fortunate that the probabilities for separate stages of the events factorize in some well-motivated approximations. This is akin to the "adiabatic approximation", where e.g. if the support of a rigid pendulum is moving at a frequency much lower than the natural frequency of the pendulum, the two motions can be treated independently or in a "factorised" way. We will not examine these stages in detail here: instead, we illustrate a possible, and common, factorisation of an event with the help of schematic diagrams as performed by a generic event generator when producing full event simulation. Figs. 3 to 7 demonstrate the 


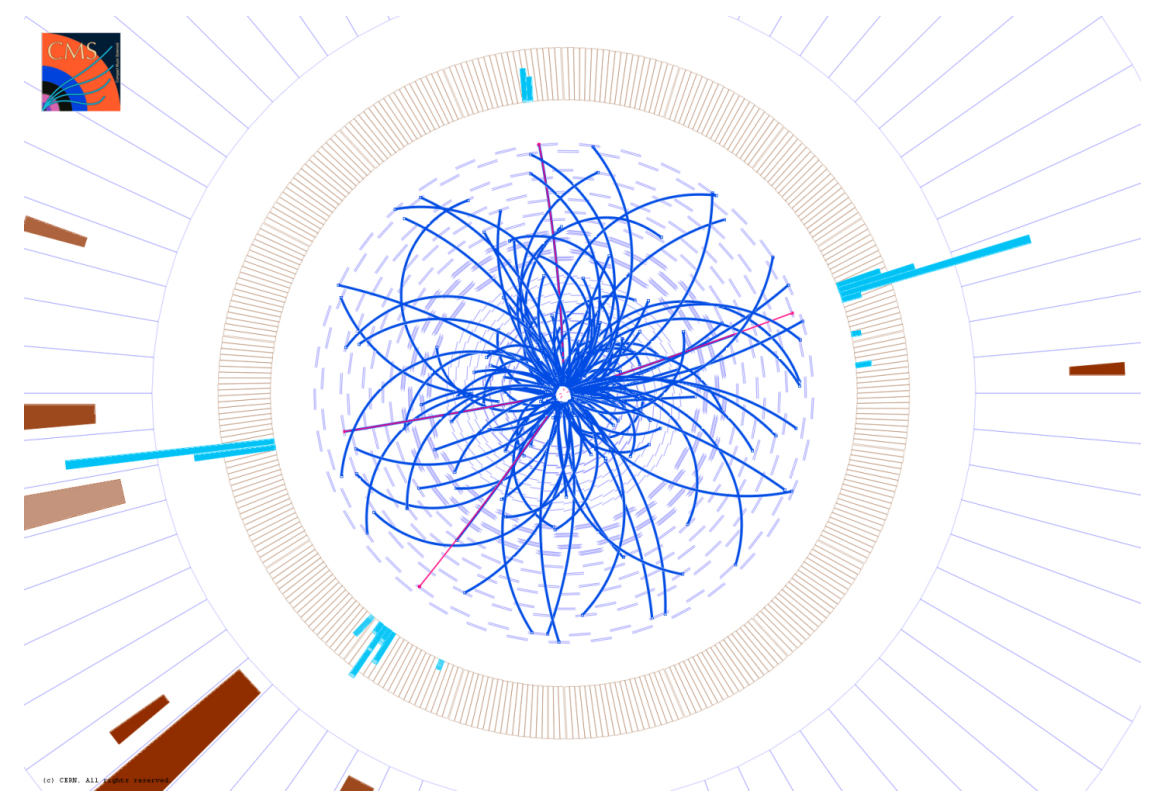

Figure 2: Real CMS proton-proton collision events in which four high energy electrons are observed. The event shows characteristics expected from the decay of a Higgs boson but is also consistent with background Standard Model physics processes.

various steps [18]. In each step, the newly appearing features are highlighted in red. In the present article we will only examine how step 1 is implemented in a numerical simulation.

1. Hard process generation, Figure 3: The hard process is generated by choosing a point on the phase space according to the 'hit-or-miss' method.

2. Heavy resonance decay, Figure 4: Heavy resonances with narrow widths are decayed before the parton shower. In this example the heavy resonance could be a top quark, decaying to a $\ell \nu_{\ell}$ and a $b$-quark.

3. Parton showers, Figure 5: The incoming partons are showered by "evolving backwards" to the incoming hadrons, producing initial-state radiation. Any final-state particles that are colour-charged also radiate, producing final-state radiation.

4. Multiple parton interactions, Figure 6: Secondary, lower-energy interactions between partons within the colliding hadrons, modelled as QCD $2 \rightarrow 2$ interactions, are generated.

5. Hadronization and hadron decays, Figure 7: In the cluster model of hadronization, clusters of coloured (QCD-charged) particles are formed and hadrons are produced. Unstable hadrons are subsequently decayed. 


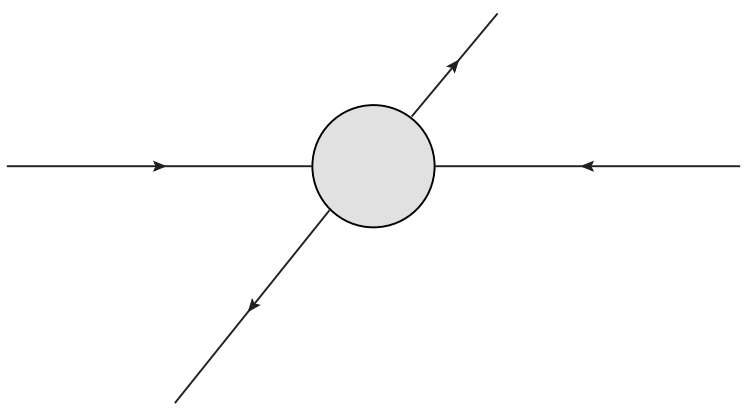

Figure 3: STEP 1: Generation of the hard process.

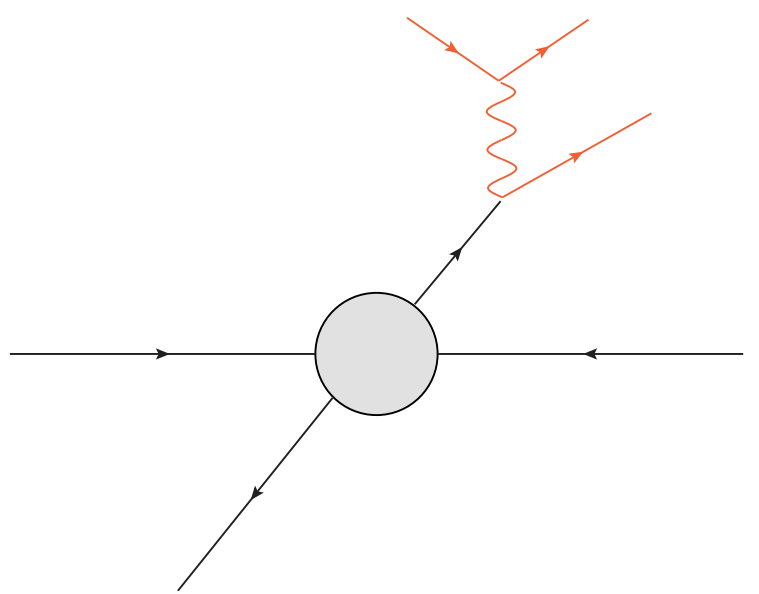

Figure 4: STEP 2: Decay of heavy resonances.

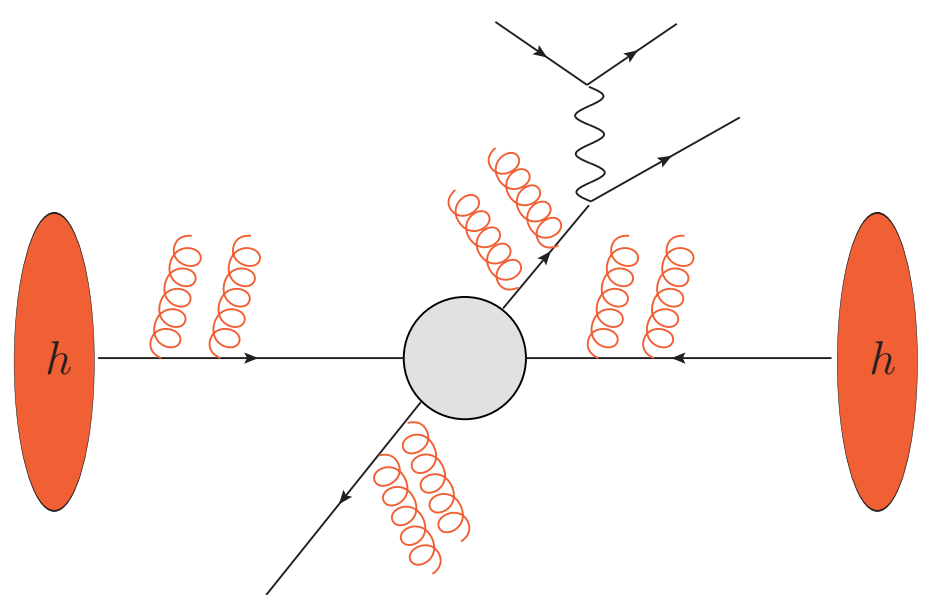

Figure 5: STEP 3: Parton showers.

\section{Exercises}

The exercises and solutions can be found at: https://apapaefs.web.cern.ch/apapaefs/ mchowto.html and are also attached to this document's source. We first review the necessary particle physics input and consider two exercises: the Monte Carlo simulation of 


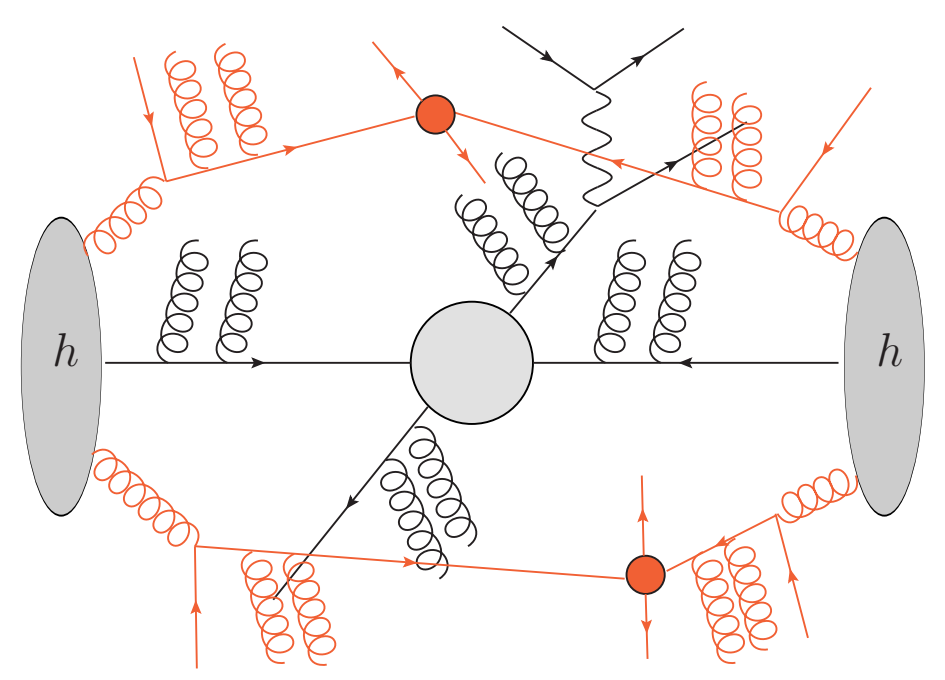

Figure 6: STEP 4: Multiple parton interactions.

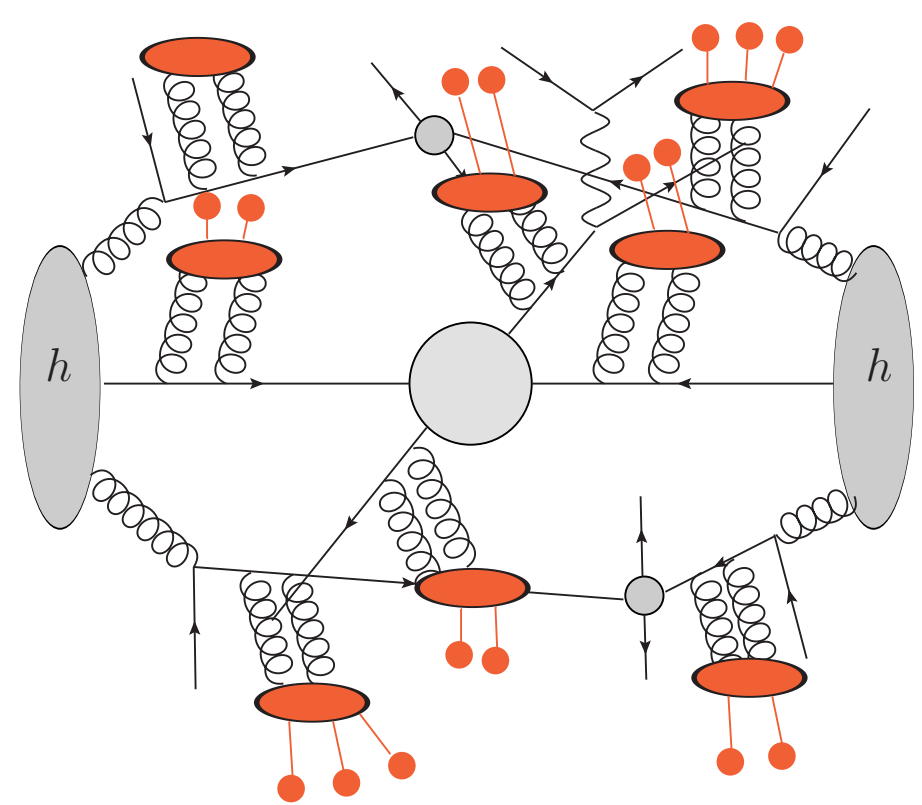

Figure 7: STEP 4: Hadronization and hadron decays.

the $e^{+} e^{-} \rightarrow \gamma \rightarrow \mu^{+} \mu^{-}$process at lepton colliders and of $q \bar{q} \rightarrow Z / \gamma \rightarrow \mu^{+} \mu^{-}$at hadron colliders.

\subsection{Particle physics input}

We first provide some basic formulae that we will employ in the exercises given in this section.

\subsection{1 $e^{+} e^{-} \rightarrow \gamma \rightarrow \mu^{+} \mu^{-}$}

The steps for calculating the matrix element and hence differential cross section for this process are given, for example, in Ref. [19], Ch. 5. Here we list the main steps in the 
calculation of $e^{+} e^{-} \rightarrow \mu^{+} \mu^{-}$in QED via photon exchange. The Feynman diagram for this process is shown in Fig. 8. Using the QED Feynman rules, one can immediately write down

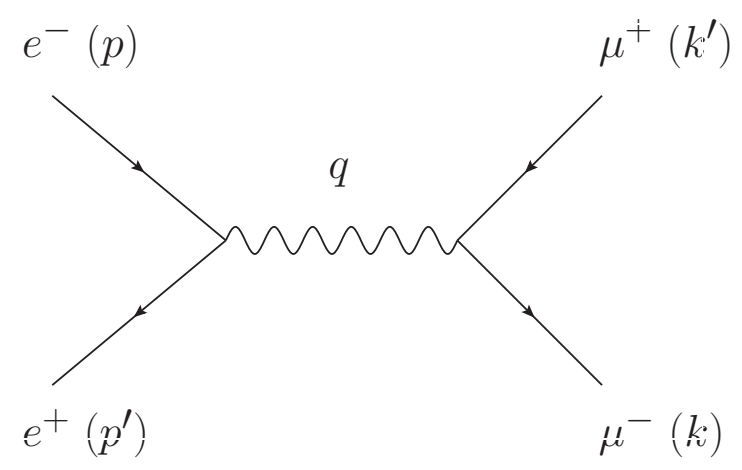

Figure 8: Feynman diagram for $e^{+} e^{-} \rightarrow \mu^{+} \mu^{-}$in QED via photon exchange.

the amplitude:

$$
i \mathcal{M}=\bar{v}^{s^{\prime}}\left(p^{\prime}\right)\left(-i e \gamma^{\lambda}\right) u^{s}(p)\left(\frac{-i g_{\lambda \nu}}{q^{2}}\right) \bar{u}^{r}(k)\left(-i e \gamma^{\nu}\right) v^{r^{\prime}}\left(k^{\prime}\right)
$$

where $s, s^{\prime}, r, r^{\prime}$ are the spin indices. Writing them implicitly, the squared matrix element is given by

$$
|\mathcal{M}|^{2}=\frac{e^{4}}{q^{4}}\left(\bar{v}\left(p^{\prime}\right) \gamma^{\lambda} u(p) \bar{u}(p) \gamma^{\nu} v\left(p^{\prime}\right)\right)\left(\bar{u}(k) \gamma_{\lambda} v\left(k^{\prime}\right) \bar{v}\left(k^{\prime}\right) \gamma_{\nu} u(k)\right)
$$

For simplicity, we can average over the electron and positron spins and sum over the muon spins:

$$
\frac{1}{2} \sum_{s} \frac{1}{2} \sum_{s^{\prime}} \sum_{r} \sum_{r^{\prime}}|\mathcal{M}|^{2} .
$$

Using completeness relations for the spinors we can write:

$$
\frac{1}{4} \sum_{\text {spins }}=\frac{e^{4}}{4 q^{4}} \operatorname{Tr}\left[\not p^{\prime} \gamma^{\lambda} \not p \gamma^{\nu}\right] \operatorname{Tr}\left[\not k \gamma_{\lambda} \not k^{\prime} \gamma_{\nu}\right],
$$

where we have neglected both the electron and muon masses. Using identities of traces of gamma matrices, one can show that:

$$
\frac{1}{4} \sum_{\text {spins }}=\frac{8 e^{4}}{q^{4}}\left[(p \cdot k)\left(p^{\prime} \cdot k^{\prime}\right)+\left(p \cdot k^{\prime}\right)\left(p^{\prime} \cdot k\right)\right] .
$$

Up to this point the matrix element squared is expressed in terms of invariant dot products. To obtain a more explicit formula we must specialise to a particular frame of reference and write down expressions for the four-vectors of the particles involved in the collision. These are shown in Fig. 9. Using the four-vector explicit expressions we can 


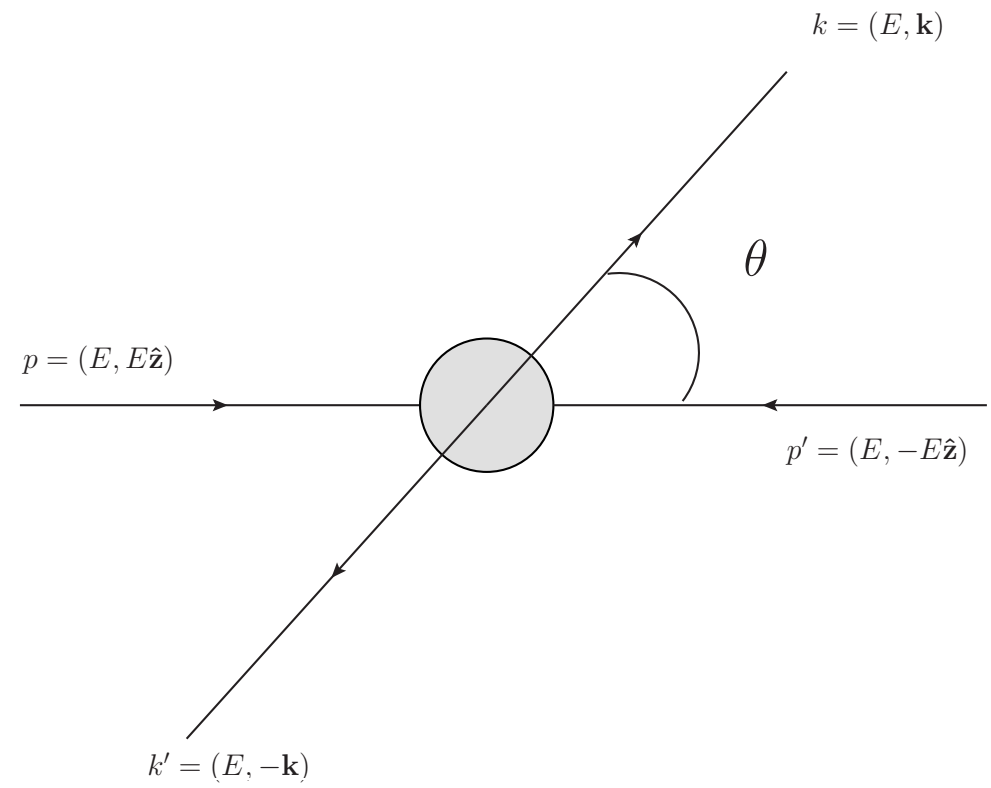

Figure 9: Schematic diagram for the kinematic setup of the process $e^{+} e^{-} \rightarrow \mu^{+} \mu^{-}$. The angle $\theta$ is defined between the incoming electron and the outgoing muon, both being particles.

express the invariants as:

$$
\begin{aligned}
q^{2}=\left(p+p^{\prime}\right)=4 E^{2}, & p \cdot p^{\prime}=2 E^{2}, \\
p \cdot k=p^{\prime} \cdot k^{\prime}=E^{2}-E|\mathbf{k}| \cos \theta, & p \cdot k^{\prime}=p^{\prime} \cdot k=E^{2}+E|\mathbf{k}| \cos \theta,
\end{aligned}
$$

where the angle $\theta$ is defined in the figure. At high enough energies we can neglect the lepton masses, $E=|\mathbf{k}|$ and:

$$
\frac{1}{4} \sum_{\text {spins }}|\mathcal{M}|^{2}=e^{4}\left(1+\cos ^{2} \theta\right)
$$

One can immediately plug the above expression into the relevant formula for the differential cross section for $2 \rightarrow 2$ scattering:

$$
\frac{\mathrm{d} \sigma}{\mathrm{d} \Omega}=\frac{1}{2 E_{\mathcal{A}} 2 E_{\mathcal{B}}\left|v_{\mathcal{A}}-v_{\mathcal{B}}\right|} \frac{|\mathbf{k}|}{(2 \pi)^{2} 4 E_{\mathrm{cm}}}|\mathcal{M}|^{2},
$$

where $E_{\mathrm{cm}}$ is the centre of mass energy of the colliding particles, the difference $\left|v_{\mathcal{A}}-v_{\mathcal{B}}\right|$ is the relative velocity of the beams as viewed from the laboratory frame, $E_{\mathcal{A}}, E_{\mathcal{B}}$ their energies in that frame and $\mathrm{d} \Omega=\mathrm{d} \cos \theta \mathrm{d} \phi$ is the phase space factor. The result is then:

$$
\frac{\mathrm{d} \sigma}{\mathrm{d} \Omega}=\frac{\alpha^{2}}{4 \hat{s}}\left(1+\cos ^{2} \theta\right),
$$

where $\alpha=e^{2} /(4 \pi)$ is the QED running coupling. Since the expression does not depend on the angle $\phi$, we may integrate over it: this introduces a multiplicative factor of $2 \pi$ on the RHS. We have also defined, $\hat{s} \equiv E_{\mathrm{cm}}^{2}$. 


\subsection{2 $e^{+} e^{-} \rightarrow Z / \gamma \rightarrow \mu^{+} \mu^{-}$}

The differential cross section for electroweak production of $\mu^{+} \mu^{-}$at a lepton collider proceeds in much the same way as the one in QED. The main difference arises from the fact that the $Z$ boson couples with different strengths to left- and right-handed fermions [20]. Table 1 shows the couplings of fermions to the $Z$ boson, in the form:

$$
\mathcal{L}_{f f Z}=-\frac{g_{W}}{2 \cos \theta_{W}} \sum_{f} \bar{\psi}_{f} \gamma^{\mu}\left(V_{f}-A_{f} \gamma_{5}\right) \psi_{f} Z_{\mu}
$$

where $g_{W}$ is the $\mathrm{SU}(2)$ coupling constant in the standard model, $\cos \theta_{W}$ is the cosine of the Weinberg angle, numerical values of which are found in Appendix A, $\psi_{f}$ represents fermion $f$ and $Z_{\mu}$ is the $Z$ boson field strength. The difference is manifested in the resulting outgoing

\begin{tabular}{llll}
\hline fermions & $Q_{f}$ & $V_{f}$ & $A_{f}$ \\
\hline $\mathrm{u}, \mathrm{c}, \mathrm{t}$ & $+\frac{2}{3}$ & $\left(+\frac{1}{2}-\frac{4}{3} \sin ^{2} \theta_{W}\right)$ & $+\frac{1}{2}$ \\
$\mathrm{~d}, \mathrm{~s}, \mathrm{~b}$ & $-\frac{1}{3}$ & $\left(-\frac{1}{2}-\frac{2}{3} \sin ^{2} \theta_{W}\right)$ & $-\frac{1}{2}$ \\
$\nu_{e}, \nu_{\mu}, \nu_{\tau}$ & 0 & $\frac{1}{2}$ & $+\frac{1}{2}$ \\
$e, \mu, \tau$ & -1 & $\left(-\frac{1}{2}+2 \sin ^{2} \theta_{W}\right)$ & $-\frac{1}{2}$ \\
\hline
\end{tabular}

Table 1: Couplings of fermions to the $Z$ boson, taken from Ref. [21].

lepton distributions as an asymmetry between the forward and backward directions. While Eq. 3.9 contains only constant terms and terms proportional to the square of the cosine of the scattering angle, the inclusion of the $Z$ boson induces a term linear in $\cos \theta$ :

$$
\frac{\mathrm{d} \sigma}{\mathrm{d} \Omega}=\frac{\alpha^{2}}{4 \hat{s}}\left[A_{0}\left(1+\cos ^{2} \theta\right)+A_{1} \cos \theta\right],
$$

where $A_{0}$ and $A_{1}$ are given by:

$$
\begin{aligned}
& A_{0}=Q_{f}^{2}-2 Q_{f} V_{\mu} V_{f} \chi_{1}+\left(A_{\mu}^{2}+V_{\mu}^{2}\right)\left(A_{f}^{2}+V_{f}^{2}\right) \chi_{2}, \\
& A_{1}=-4 Q_{f} A_{\mu} A_{f} \chi_{1}+8 A_{\mu} V_{\mu} A_{f} V_{f} \chi_{2},
\end{aligned}
$$

where in turn, the functions $\chi_{1}$ and $\chi_{2}$ are given by:

$$
\begin{aligned}
\chi_{1}(\hat{s}) & =\kappa \hat{s}\left(\hat{s}-M_{Z}^{2}\right) /\left(\left(\hat{s}-M_{Z}^{2}\right)^{2}+\Gamma_{Z}^{2} M_{Z}^{2}\right), \\
\chi_{2}(\hat{s}) & =\kappa^{2} \hat{s}^{2} /\left(\left(\hat{s}-M_{Z}^{2}\right)^{2}+\Gamma_{Z}^{2} M_{Z}^{2}\right), \\
\kappa & =\sqrt{2} G_{f} M_{Z}^{2} /(4 \pi \alpha) .
\end{aligned}
$$

A good test to check whether the Monte Carlo integration is working is to check whether the Monte Carlo cross section agrees with the analytic result:

$$
\sigma=\frac{4 \pi \alpha^{2}}{3 \hat{s}} A_{0},
$$

where it is evident that the $\cos \theta$ term has dropped out due to its asymmetry. 


\subsection{Exercise 1: lepton colliders}

In this exercise the aim is to produce a Monte Carlo event generator for $e^{+} e^{-} \rightarrow Z / \gamma \rightarrow$ $\mu^{+} \mu^{-}$. Of course the choice of 'final' flavour is arbitrary, since we have neglected all lepton masses to this point. Note, however, that if one wants to consider $e^{+} e^{-} \rightarrow e^{+} e^{-}$, then there exists a new $t$-channel diagram that is not included in the above expression.

The integration to obtain the cross section is in fact trivial, since we know how to integrate cosine functions analytically, and the $e^{+} e^{-}$centre-of-mass energy, $\hat{s}$, is fixed, without requiring any Jacobian transformations to improve efficiency (i.e. there's no $\mathrm{d} m^{2}$ integral as in Eq. 2.8). Nevertheless, the exercise provides an insight to the basic building blocks of an event generator. The algorithm is given in Section 2.3. One thing to notice is that to obtain the cross sections in picobarn, one has to use the conversion factor in Table 2 in Appendix A.

The example 'solution' was written in Python, and provides some basic plotting using Matplotlib. A histogram of the only variable $\cos \theta$ is given. In this case this is an observable that we can measure, since we know both the direction of the incoming lepton and the outgoing lepton (between which this angle is defined). Moreover, the momenta are 'set up' in the laboratory frame, which is equivalent to the centre-of-mass frame in this case.

Some suggestions for possible extensions:

- Check the cross section against the analytical formula. For example, at $E_{\mathrm{cm}}=90 \mathrm{GeV}$ : $\sigma=1060.82 \pm 0.25 \mathrm{pb}$ versus the analytic result: $\sigma_{\text {analytic }}=1060.93 \mathrm{pb}$.

- Plot distributions of the energy of particles, or the pseudo-rapidity (in this case equal to the rapidity since we neglect the mass): $\eta=-\ln \tan (\theta / 2)$.

- Investigate the forward-backward asymmetry: $A_{\mathrm{FB}} \equiv\left(\sigma_{F}-\sigma_{B}\right) /\left(\sigma_{F}+\sigma_{B}\right)$, where $\sigma_{F, B}$ are the forward (right 'hemisphere', $\theta \in(-\pi / 2,+\pi / 2)$ ) and backward (left 'hemisphere', $\theta \in(\pi / 2,+\pi) \cup(-\pi / 2,-\pi))$ cross sections respectively.

\subsection{Exercise 2: hadron colliders}

The previous exercise involved essentially a one-dimensional integral, over the angle $\theta$. For an electron-positron collision, this is always the case for a $2 \rightarrow 2$ hard process. The next incremental complication arises for hard processes at hadron colliders. Since the hadrons are not elementary particles, we have to consider collisions between their constituent quark and gluons (partons), at high enough energies $(E \gg 1 \mathrm{GeV})$. This results in the following considerations:

- The centre-of-mass energy of the colliding partons is not fixed, i.e. $\hat{s}$ is variable. Moreover, since the centre-of-mass frame and the laboratory frame (where observations are made) are not the same, the final-state particles need to be Lorentz-boosted from one frame to the other, in order to construct observable distributions.

- We need to consider the distribution of momenta of the colliding partons inside the protons as well as the different contributing quark flavours, characterised by the parton density functions. The parton density function for flavour $q$ for a quark (or gluon) 
carrying momentum fraction $x$ of the proton at momentum transfers $Q^{2}$ is denoted by $f_{q}\left(x, Q^{2}\right)$. This can be accessed via the LHAPDF library [22, 23]. For more details on the LHAPDF interface, see Appendix B.

- Due to the above two points, we now have essentially four variables that characterise the phase space: $\hat{s}$, the momentum fractions $x_{1,2}$ and the scattering angle $\theta$, plus one constraint allowing us to eliminate one: $\hat{s}=x_{1} x_{2} S$, where $S$ is the proton-proton centre of mass energy squared. This leaves us with a 3-dimensional phase space for the hard process at hadron colliders.

- When summing over quark flavours, one has to note that the angle $\cos \theta$ is defined with respect to the incoming particle (as opposed to anti-particle) and the outgoing particle (as opposed to anti-particle). This implies that for example, in a collision of $u \bar{u}$, if $\theta$ is defined with respect to the positive $z$-axis, one must add a contribution for $\bar{u} u$, with $\theta \rightarrow \pi-\theta$, resulting in the change $\cos \theta \rightarrow-\cos \theta$. Effectively this cancels out the asymmetric part of the distribution in a proton-proton collider (but not in a $p \bar{p}$ collider such as the Tevatron).

- For the purposes of this exercise we will cut-off the di-lepton invariant mass at some value, $Q_{\mathrm{min}}$. This will appear in the limits of the integrals we perform. For reasonable results, we will choose $Q_{\min }=60 \mathrm{GeV}$.

- The matrix element squared has to be multiplied by a factor of $1 / 3$ : this averages over the initial quark-anti-quark colour configurations. If we also had quarks in the final state, we would need to sum over their colours.

The partonic cross section of Eq. 3.11 is still valid in the case of $q \bar{q} \rightarrow Z / \gamma \rightarrow \mu^{+} \mu^{-}$, with the quark charges taken into consideration accordingly. However, we must now consider the hadronic cross section:

$$
\frac{\mathrm{d} \sigma}{\mathrm{d} \hat{s} \mathrm{~d} \cos \theta}=\sum_{q, q^{\prime}} \int_{0}^{1} \mathrm{~d} x_{1} \int_{0}^{1} \mathrm{~d} x_{2} \delta\left(\hat{s}-x_{1} x_{2} S\right) f_{q}\left(x_{1}, \hat{s}\right) f_{q^{\prime}}\left(x_{2}, \hat{s}\right) \frac{\mathrm{d} \hat{\sigma}}{\mathrm{d} \cos \theta},
$$

with $\mathrm{d} \hat{\sigma} / \mathrm{d} \cos \theta$ given by Eq. 3.11, and we have already made the replacement $Q^{2}=\hat{s}$ for the PDF factorisation scale. The sum is written here generically, over $q$ and $q^{\prime}$ but should be taken over $q \bar{q}$ for the process we are considering. The integral over the $\delta$-function can then be performed to eliminate one of the dependent observables. We remove $x_{2}$ and remove the integral over $x_{1}$, turning it into a differential on the left-hand side:

$$
\begin{aligned}
\frac{\mathrm{d} \sigma}{\mathrm{d} \hat{s} \mathrm{~d} x_{1} \mathrm{~d} \cos \theta} & =\int_{0}^{1} \mathrm{~d} x_{2} \delta\left(S x_{1}\left(x_{2}-\hat{s} /\left(S x_{1}\right)\right) f_{q}\left(x_{1}, \hat{s}\right) f_{q^{\prime}}\left(x_{2}, \hat{s}\right) \frac{\mathrm{d} \hat{\sigma}}{\mathrm{d} \cos \theta},\right. \\
& =\frac{1}{\hat{s} x_{1}} f_{q}\left(x_{1}, \hat{s}\right) f_{q^{\prime}}\left(x_{2}=\hat{s} /\left(S x_{1}\right), \hat{s}\right) \frac{\mathrm{d} \hat{\sigma}}{\mathrm{d} \cos \theta} .
\end{aligned}
$$

We define $\tau \equiv \hat{s} / S$ and the rapidity of the outgoing di-lepton system:

$$
y \equiv \frac{1}{2} \ln \left(\frac{E+p_{z}}{E-p_{z}}\right)=\frac{1}{2} \ln \left(\frac{x_{1}}{x_{2}}\right),
$$


by which $x_{1,2}=\sqrt{\tau} \mathrm{e}^{ \pm y}$, and:

$$
\mathrm{d} x_{1} \mathrm{~d} \hat{s} /\left(\hat{s} x_{1}\right)=\mathrm{d} \tau \mathrm{dy}
$$

We finally arrive at:

$$
\frac{\mathrm{d} \sigma}{\mathrm{d} \tau \mathrm{d} y \mathrm{~d} \cos \theta}=\sum_{q, q^{\prime}} f_{q}\left(x_{1}=\sqrt{\tau} \mathrm{e}^{+y}, \hat{s}=\tau S\right) f_{q^{\prime}}\left(x_{2}=\sqrt{\tau} \mathrm{e}^{-y}, \hat{s}=\tau S\right) \frac{\mathrm{d} \hat{\sigma}}{\mathrm{d} \cos \theta} .
$$

The integration over the phase space can be performed via the Monte Carlo method by selecting $\tau, y$ and $\cos \theta$ randomly. Since we know we have a heavy resonance (the $Z$ boson) in the process, we can attempt to perform a Jacobian transformation as was described in Section 2.2. Note, however, that in this case the phase space is not flat after transformation since we have the photon contribution at low invariant masses, as well as the interference contribution. Nevertheless, the transformation is still useful and it is recommended. One can experiment with the parameters of the transformation relation to see if the variance can be decreased by clever choices. Hence, for random numbers $R_{i} \in(0,1), i=1,2$ :

$$
\begin{aligned}
\cos \theta & =2 R_{1}-1 \\
y & =\left(2 R_{2}-1\right) y_{\max },
\end{aligned}
$$

with the maximum value of the rapidity given by: $y_{\max }=-0.5 \ln (\tau)$. The $\tau$-integral has to be more carefully considered. Defining the transform mass and width parameters $M_{\mathrm{tr}}$ and $\Gamma_{\mathrm{tr}}$, respectively, and keeping them free for the moment, we have:

$$
\tau S=\hat{s}=M_{\mathrm{tr}} \Gamma_{t r} \tan (\rho)+M_{\mathrm{tr}}^{2}
$$

with $\rho$ in $\left(\rho_{\min }, \rho_{\max }\right)$, generated using random number $R_{3} \in(0,1)$ via:

$$
\rho=\rho_{\min }+\left(\rho_{\max }-\rho_{\min }\right) R_{3},
$$

where $\rho$ is limited by the choice of $Q_{\min }$ and the hadron centre-of-mass energy $\sqrt{S}$ :

$$
\begin{gathered}
\rho_{\min }=\tan ^{-1}\left(\frac{Q_{\min }^{2}-M_{\mathrm{tr}}^{2}}{\Gamma_{\mathrm{tr}} M_{\mathrm{tr}}}\right), \\
\rho_{\max }=\tan ^{-1}\left(\frac{S-M_{\mathrm{tr}}^{2}}{\Gamma_{\mathrm{tr}} M_{\mathrm{tr}}}\right) .
\end{gathered}
$$

The integration can be performed in an equivalent way as in Exercise 1, and the maximum weight can be stored to perform the 'hit-or-miss' unweighing of events. This is again, exactly equivalent to the case of lepton colliders. A final complication for the case of the hard processes at hadron colliders is boosting between the centre-of-mass frame (where the calculation of the partonic cross section was performed) into the lab frame. We already know the 4-momenta in the lab frame for the incoming partons:

$$
\begin{aligned}
& p_{q}^{\mathrm{lab}}=\frac{\sqrt{\hat{s}}}{2}\left(x_{1}, 0,0, x_{1}\right) \\
& p_{q^{\prime}}^{\mathrm{lab}}=\frac{\sqrt{\hat{s}}}{2}\left(x_{2}, 0,0,-x_{2}\right),
\end{aligned}
$$


where $\sqrt{\hat{s}}=E_{\mathrm{cm}}$ is the centre-of-mass frame collision energy of the partons. The Lorentz boost factor along the $z$-axis between the lab and centre-of-mass frames can be calculated and is given by:

$$
\beta=\frac{x_{2}-x_{1}}{x_{2}+x_{1}}
$$

where $\beta=v / c$. And hence, the momenta in the centre-of-mass frame:

$$
\begin{aligned}
& p_{\mu}^{\mathrm{cm}}=\frac{\sqrt{\hat{s}}}{2}(1, \sin \theta \cos \phi, \sin \theta \sin \phi, \cos \theta) \\
& p_{\bar{\mu}}^{\mathrm{cm}}=\frac{\sqrt{\hat{s}}}{2}(1,-\sin \theta \cos \phi,-\sin \theta \sin \phi,-\cos \theta),
\end{aligned}
$$

(where $\phi$ has been generated randomly and uniformly using a random number $R_{4} \in(0,1)$ : $\left.\phi=2 \pi R_{4}\right)$ can be transformed into those in the lab frame via a Lorentz boost along the $z$-direction:

$$
p^{\mathrm{lab}}=\left(\gamma p_{0}-\gamma \beta p_{3}, p_{1}, p_{2},-\gamma \beta p_{0}+\gamma p_{3}\right),
$$

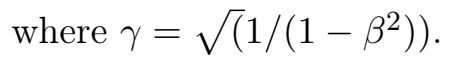

The solution to this exercise is provided as a Python program as well, and generates a set of histograms using the Matplotlib library.

Some suggestions for further investigations:

- Calculate the cross sections for di-lepton production via $Z \gamma$ at proton-proton colliders $8 \mathrm{TeV}$ and $14 \mathrm{TeV}$ using the cteq611 PDF sets and compare to the MadGraph results: $\sigma(8 \mathrm{TeV})=(881.8 \pm 1) \mathrm{pb}$ and $\sigma(14 \mathrm{TeV})=(1684 \pm 1.3) \mathrm{pb}$. Note that the minimum same-flavour lepton invariant mass was taken to be $60 \mathrm{GeV}$ and no other cuts were imposed on the leptons.

- Consider the modifications necessary to simulate a $p \bar{p}$ collider.

- The Les Houches file format allows one to write parton-level events and feed them into a general-purpose Monte Carlo for parton showering and hadronization. An explanation of how the format looks like is found in Appendix C.

\section{After the hard process}

Even though we will not go into the technical details of the implementation of the following steps in event generation, it is interesting to list some of the considerations necessary to perform them. The factorised view of Monte Carlo event generation has already been illustrated by Figs. 3 to 7. Step-by-step, some points that need to be considered are:

1. The hard process can be $2 \rightarrow N$, where $N$ is any number of particles.

2. Decays can be easily implemented on top of any process in a factorised way, given that the resonance is narrow enough. If this is the case, one can consider the decay of a massive resonance in its rest frame, and then boost the decay products into the lab frame according to the particle's boost in that frame. 
3. Most parton showers are based on collinear and soft splitting kernels that capture the enhanced regions. There are two possibilities for parton showers, with some technical differences in the implementation: radiation from final-state particles or radiation from initial-particles. The difference arises because initial-state particles need to 'evolve' back to the incoming hadrons, whereas final-state particles have to evolve forward to hadrons.

4. At some scale, $\mathcal{O}(1 \mathrm{GeV})$, perturbation theory breaks down and a non-perturbative model needs to take over. The phenomenon is called hadronization. The outgoing quarks and gluons need to be treated through some model that groups them into QCD colour-singlets. This is done in HERWIG 7, for example, via a cluster model, and in Pythia 8 via a string model. Another non-perturbative effect involves the interaction of multiple partons. In HERWIG 7 and Sherpa this phenomenon modelled as multiple QCD $2 \rightarrow 2$ interactions [14, 24]. In Pythia 8 it is treated as being formed through interleaved parton-parton interactions in a common sequence with the initial-state radiation [25].

\section{Conclusions}

We have presented a short introduction to Monte Carlo event generators and directly delved into two simple examples. Solutions to the exercises are given and motivations on how one can go beyond were presented.

\section{Acknowledgements}

The author would like to thank Christoph Grab and Nicolas Chanon the opportunity to lecture at the "Advanced Scientific Computing Workshop" at ETH Zürich, as well the students who attended the course, providing helpful feedback. Support is acknowledged in part by the Swiss National Science Foundation (SNF) under contract 200020-149517, by the European Commission through the "LHCPhenoNet" Initial Training Network PITN-GA2010-264564, MCnetITN FP7 Marie Curie Initial Training Network PITN-GA-2012-315877 and by a Marie Curie Intra European Fellowship within the 7th European Community Framework Programme (grant no. PIEF-GA-2013-622071).

\section{A Constants}

The constants in this section are given to provide agreement with the MadGraph event generator. They appear in Table 2.

\section{B Parton density functions using LHAPDF}

At the time of writing, the latest version of the LHAPDF package is 6.1.3. It is recommended to use this or a latter version for the exercises given here. The library can be interfaced to either C++, FORTRAN or Python. Since the solutions to the exercises are given in Python, the PDFs should be initialised as: 


\begin{tabular}{lll}
\hline variable & symbol & value \\
\hline conversion factor & $\mathrm{GeV}^{-2} \rightarrow \mathrm{pb}$ & $3.894 \times 10^{8}{\mathrm{pb} \mathrm{per} \mathrm{GeV}^{-2}}$ \\
$Z$ boson mass & $M_{Z}$ & $91.188 \mathrm{GeV}$ \\
$Z$ boson width & $\Gamma_{Z}$ & $2.4414 \mathrm{GeV}$ \\
QED running coupling & $\alpha$ & $\frac{1}{132.507}$ \\
Fermi constant & $G_{f}$ & $1.16639 \times 10^{-5} \mathrm{GeV}^{-2}$. \\
Weinberg angle & $\sin ^{2} \theta_{W}$ & 0.222246 \\
\hline
\end{tabular}

Table 2: Constants used throughout this article, given to provide agreement with MadGraph.

\#\# import LHAPDF and initialise PDFs

import lhapdf

\#\# initialises PDF member object (for protons)

$\mathrm{p}=$ lhapdf.mkPDF ("cteq611", 0 )

and the PDF should be called as:

$\mathrm{p} . \mathrm{xfxQ}(\mathrm{FLAVOUR}, \mathrm{x} 1, \mathrm{mu})$

where FLAVOUR should be replaced by the quark flavours contributing to the process: 1 for down-quarks, 2 for up, 3 for strange, 4 for charm and negative values for the corresponding anti-quarks. The gluon, not used here, is given by 21 . Note that this actually gives $x \times f(x)$ and thus one has to divide by the momentum fraction to get $f(x)$. Moreover, this specific function takes as input the scale and not the scale squared.

\section{The Les Houches event file format}

The file header and the first event in a Les Houches-accord event file have the following form:

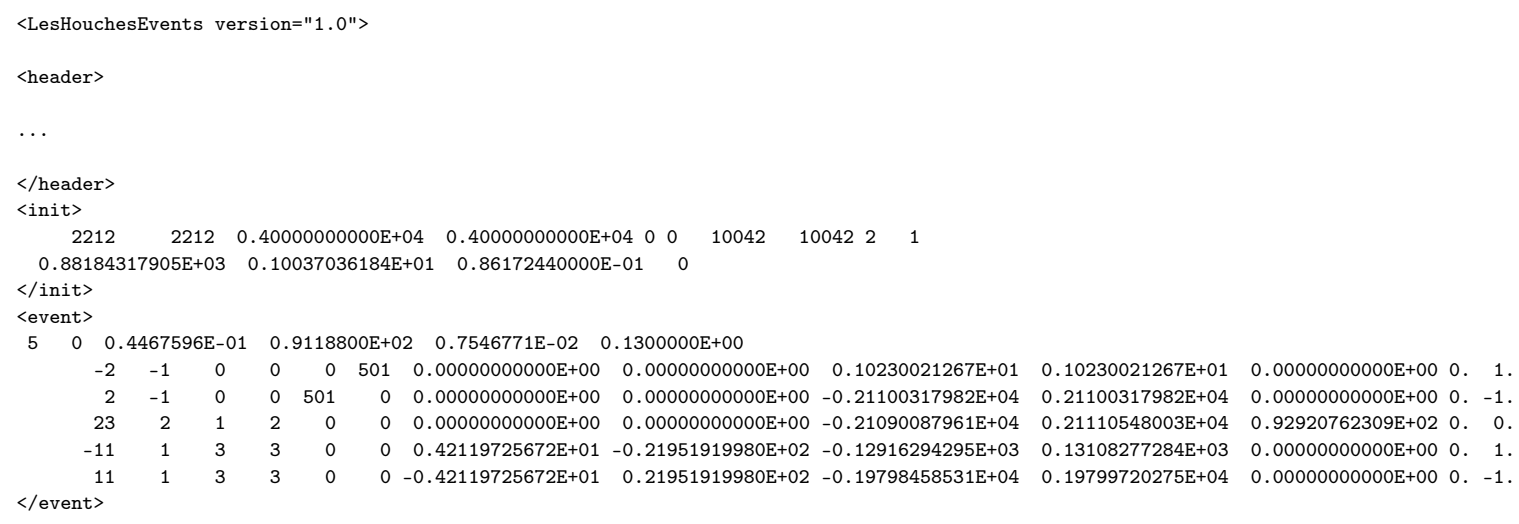


The first row after <init> shows the ids of the incoming hadrons, their energy and the PDF numbers (10042 in this case). The following line shows the cross section and the error. The first event follows, containing the particle ids, their status codes and mother information, colour information, their momenta, and whether they are stable particles or not. See Ref. [26] for more details.

\section{Convergence}

\begin{tabular}{lll}
\hline Technique & Convergence & worse than $\mathrm{MC}$ in d $>$ \\
\hline trapezium & $1 / N^{2 / d}$ & 4 \\
Simpson's & $1 / N^{4 / d}$ & 8 \\
$m$ th-order gaussian quadra- & $1 / N^{(2 m-1) / d}$ & $4 m-2$ \\
ture & & \\
Monte Carlo & $1 / \sqrt{N}$ & - \\
\hline
\end{tabular}

Table 3: The rate of convergence with the number of points $N$ used for each method in $d$-dimensions.

\section{References}

[1] Wikipedia. How-to. https://en.wikipedia.org/wiki/How-to, 2020.

[2] M. Twain. Following the Equator. Sun-Times Media Group, 1897.

[3] Johan Alwall, Michel Herquet, Fabio Maltoni, Olivier Mattelaer, and Tim Stelzer. MadGraph 5 : Going Beyond. JHEP, 06:128, 2011. doi: 10.1007/JHEP06(2011)128.

[4] J. Alwall, R. Frederix, S. Frixione, V. Hirschi, F. Maltoni, O. Mattelaer, H. S. Shao, T. Stelzer, P. Torrielli, and M. Zaro. The automated computation of tree-level and next-to-leading order differential cross sections, and their matching to parton shower simulations. JHEP, 07:079, 2014. doi: 10.1007/JHEP07(2014)079.

[5] M. Bahr et al. Herwig++ Physics and Manual. Eur. Phys. J., C58:639-707, 2008. doi: 10.1140/epjc/s10052-008-0798-9.

[6] K. Arnold et al. Herwig ++ 2.6 Release Note. 2012.

[7] S. Gieseke et al. Herwig ++2.5 Release Note. 2011.

[8] Johannes Bellm et al. Herwig 7.2 Release Note. 2019.

[9] Johannes Bellm et al. Herwig 7.1 Release Note. 2017. 
[10] Johannes Bellm et al. Herwig 7.0/Herwig ++3.0 release note. Eur. Phys. J., C76(4):196, 2016. doi: $10.1140 /$ epjc/s10052-016-4018-8.

[11] J. Bellm et al. Herwig ++2.7 Release Note. 2013.

[12] Torbjorn Sjostrand, Stephen Mrenna, and Peter Z. Skands. PYTHIA 6.4 Physics and Manual. JHEP, 05:026, 2006. doi: 10.1088/1126-6708/2006/05/026.

[13] Torbjorn Sjostrand, Stephen Mrenna, and Peter Z. Skands. A Brief Introduction to PYTHIA 8.1. Comput. Phys. Commun., 178:852-867, 2008. doi: 10.1016/j.cpc.2008.01.036.

[14] T. Gleisberg, Stefan. Hoeche, F. Krauss, M. Schonherr, S. Schumann, F. Siegert, and J. Winter. Event generation with SHERPA 1.1. JHEP, 02:007, 2009. doi: 10.1088/1126-6708/2009/02/007.

[15] Andy Buckley et al. General-purpose event generators for LHC physics. Phys. Rept., 504: 145-233, 2011. doi: 10.1016/j.physrep.2011.03.005.

[16] W. Kittel, L. Van Hove, and W. Wojcik. A monte carlo generation method with importance sampling for high energy collisions of hadrons. Comput. Phys. Commun., 1:425-436, 1970. doi: 10.1016/0010-4655(70)90016-0.

[17] G.Peter Lepage. VEGAS: AN ADAPTIVE MULTIDIMENSIONAL INTEGRATION PROGRAM. 31980.

[18] Andreas Papaefstathiou. Phenomenological aspects of new physics at high energy hadron colliders. PhD thesis, Cambridge U., 2011. URL

https://www.repository.cam.ac.uk/handle/1810/239399.

[19] Michael E. Peskin and Daniel V. Schroeder. An Introduction to quantum field theory. Addison-Wesley, Reading, USA, 1995. ISBN 9780201503975, 0201503972. URL http://www.slac.stanford.edu/ mpeskin/QFT.html.

[20] F. Halzen and A. Martin. Quarks and Leptons: An Introductory Course in Modern Particle Physics. John Wilie and Sons, 1984.

[21] R. Keith Ellis, W. James Stirling, and B. R. Webber. QCD and collider physics. Camb. Monogr. Part. Phys. Nucl. Phys. Cosmol., 8:1-435, 1996.

[22] M. R. Whalley, D. Bourilkov, and R. C. Group. The Les Houches accord PDFs (LHAPDF) and LHAGLUE. In HERA and the LHC: A Workshop on the implications of HERA for LHC physics. Proceedings, Part B, pages 575-581, 2005.

[23] LHAPDF. http://lhapdf.hepforge.org/, 2020.

[24] Manuel Bahr, Stefan Gieseke, and Michael H. Seymour. Simulation of multiple partonic interactions in Herwig ++. JHEP, 07:076, 2008. doi: 10.1088/1126-6708/2008/07/076.

[25] T. Sjostrand and Peter Z. Skands. Transverse-momentum-ordered showers and interleaved multiple interactions. Eur. Phys. J. C, 39:129-154, 2005. doi: 10.1140/epjc/s2004-02084-y.

[26] Johan Alwall et al. A Standard format for Les Houches event files. Comput. Phys. Commun., 176:300-304, 2007. doi: 10.1016/j.cpc.2006.11.010. 